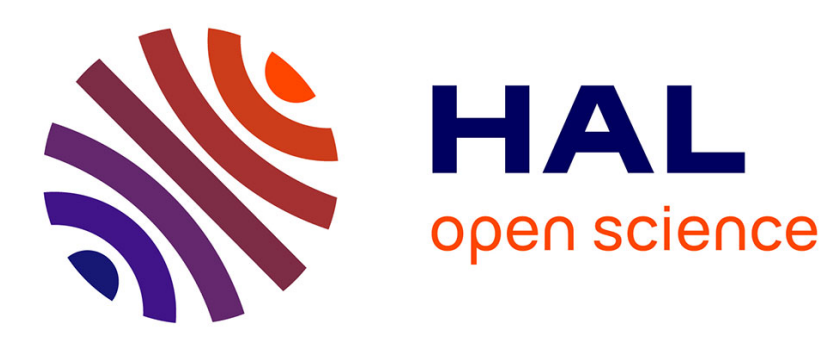

\title{
Micromagnetic model of magnetization reversal of magnetically hard ultrathin dots and stripes
}

\author{
Mustafa Eleoui, Olivier Fruchart, Jean-Christophe Toussaint
}

\section{To cite this version:}

Mustafa Eleoui, Olivier Fruchart, Jean-Christophe Toussaint. Micromagnetic model of magnetization reversal of magnetically hard ultrathin dots and stripes. Journal of Magnetism and Magnetic Materials, 2004, 283, pp.46. 10.1016/j.jmmm.2004.05.009 . hal-00004737

\section{HAL Id: hal-00004737 \\ https://hal.science/hal-00004737}

Submitted on 17 Apr 2005

HAL is a multi-disciplinary open access archive for the deposit and dissemination of scientific research documents, whether they are published or not. The documents may come from teaching and research institutions in France or abroad, or from public or private research centers.
L'archive ouverte pluridisciplinaire HAL, est destinée au dépôt et à la diffusion de documents scientifiques de niveau recherche, publiés ou non, émanant des établissements d'enseignement et de recherche français ou étrangers, des laboratoires publics ou privés. 


\title{
Micromagnetic model of magnetization reversal of magnetically hard ultrathin dots and stripes
}

\author{
M.Eleoui *, O. Fruchart, J.-C. Toussaint \\ Laboratoire Louis Néel (CNRS UPR5051), BP166, F-38042 Grenoble Cedex 9, \\ France
}

\begin{abstract}
We propose improvements and extensions to an analytical model of magnetization reversal in ultrathin flat dots and stripes with in-plane uniaxial anisotropy. Owing to the localized character of nucleation volumes in hard magnetic materials, we use the concept of edge demagnetizing torque, where all demagnetizing effects are applied at the dot's edge. The magnetization state and the reversal field $\tilde{h}_{\mathrm{r}}$ are predicted as a function of magnetization, dot thickness and in-plane edge orientation. An excellent agreement is found with numerical simulations. An approximate but accurate scaling law is proposed for an easy computation of $\tilde{h}_{\mathrm{r}}$. The model is shown to be valid for dots thinner than both exchange length and wall width, and lateral size well above each of these lengths.
\end{abstract}

Key words: Magnetization reversal, Micromagnetic modelling, Magnetic dot, Coercivity.

PACS: 75.60.Jk; 75.75.+a

\section{Introduction}

Over the last ten years many experimental studies have reported magnetization reversal processes of micron- or sub-micron-scale flat dots [1]. These studies are motivated by the development of devices requiring the use of such dots, like magnetic field sensors and magnetic random access memories (MRAMs) [2]. This has led to the outlining of the influence of parameters such as dot shape and size[3,4], edge roughness[5,6], magneto-crystalline anisotropy[7,8],

* Corresponding author.

Email address: eleoui@grenoble.cnrs.fr (M.Eleoui). 
and dot thickness[3,9]. Although numerical calculations can nowadays be performed with desktop computers, analytical modelling is still desirable for its capacity to predict rapidly general trends, in the form of power laws for example. Analytical models are mostly available for soft magnetic materials [10-13]. In a previous paper we proposed an analytical model making use of suitable approximations to describe the magnetization reversal of thin flat rectangular dots with in-plane magneto-crystalline uniaxial anisotropy, and ith applied field and edges parallel to the anisotropy axis [14]. The model is suited to investigate the ultrathin range (typically below $5 \mathrm{~nm}$ ), making it a tool to predict the impact of future scaling-down of the size of devices. In the present article we propose an improved version of this model. The first improvement consists of a more accurate evaluation of the strength of dipolar fields, reducing the discrepancy with numerical calculations by about $30 \%$. The second improvement consists of the ability to consider dots with an arbitrary orientation of the edges with the anisotropy axis and external field direction, the latter two still being assumed to be parallel.

The outline of the article is as follows. We first briefly remind the reader of the principle and results of the existing model[14], that are required to understand the new calculations. We then update the existing analytical results following the more accurate estimation of dipolar fields, and propose extended results for the new dot geometry tackled by the model. Numerical evaluation of the predicted reversal field is then given as a function of edge orientation and dot thickness. In addition to the analytical results, we propose an approximate although accurate scaling law to assist the reader in estimating without long calculations the reversal field value as a function of magnetization, exchange, dot thickness and magnetic anisotropy. Finally, we discuss the results against numerical simulations, estimate its range of validity in terms of anisotropy and thickness values, and finally describe consequences for experiments.

\section{Preliminary}

Most of this section has been explained in detail in [14]. A summary is given here to ease the independent reading of the present article.

\subsection{Principle and approximations of the model}

We wish to address dots of lateral size much larger than characteristic magnetic length scales. Thus magnetization reversal is not expected to be uniform and follow the Stoner-Wohlfarth model[15,16]. Besides, we do not consider dots made of soft magnetic material, but instead made of material with a 
non-negligible magnetic anisotropy energy of microscopic origin, like magnetocrystalline anisotropy energy. Thus, the reversal is nor expected to be collective (i.e. not involving all the spins like, e.g., for curling mode[17]). It should instead proceed via nucleation and propagation of a domain wall, like in hard magnetic materials. If we exclude the occurrence of defects, nucleation volumes are expected to be located close to some dot edge, where demagnetizing fields are the highest. It is the purpose of this paragraph to introduce physicallyrelevant approximations that allow us to describe these nucleation volumes analytically, and thus predict features of magnetization reversal. Three approximations are introduced:

(1) Magnetization lies in-plane and does not vary along the normal to the surface, even close to edges. This should be valid for thicknesses smaller than characteristic magnetic length scales, see below.

(2) As nucleation volumes are expected to grow close to edges, only edges need to be described to derive reversal field values. We also assume a translation symmetry parallel to the dot's edge, mimicking a slow variation along this direction. Thus, the geometry is simplified to a semiinfinite dot with a one-dimensional degree of freedom (Figs. 1;3), the in-plane magnetization direction $\omega(z)$ for $z \leq 0$.

(3) The obstacle against solving general micromagnetic problems is to tackle dipolar fields, that are non-local and non-linear. In our case we showed[14] that for dots thinner than magnetic length scales the effect of demagnetizing fields can be assumed to result in a torque $\Gamma_{\mathrm{d}}$ applied exactly at the edge of the dot $(z=0)$. The validity of this approximation is granted by the fact that dipolar fields are short-ranged in two dimensions, with a length scale similar to the thickness of the structure considered. Finally, only surface charges $\mathbf{M}_{\mathbf{s}} \cdot \mathbf{n}$ located at the edge are taken into account (no volume charges).

These approximations allowed us to integrate micromagnetic equations and predict the nucleation field $\tilde{h}_{\mathrm{n}}$ (first deviation from strictly uniform magnetization), then the nucleation volume profile from the edge towards the dot center, and finally the reversal field $\tilde{h}_{\mathrm{r}}$ (irreversible magnetization jump). The range of validity of the model arising from these approximations will be discussed in terms of thickness and anisotropy magnitude in section 7.3.

\subsection{Dimensionless units}

In most of the paper we make use of dimensionless units[14]. As a general rule uppercase and lowercase letters stand for dimensional and dimensionless units, respectively. We use the following relations, where $A$ is the exchange constant $\left(\mathrm{J}^{\mathrm{m}} \mathrm{m}^{-1}\right), K$ is the in-plane second-order anisotropy constant of the dot 
material $\left(\mathrm{J} . \mathrm{m}^{-3}\right), M_{\mathrm{s}}$ the volume magnetization $\left(\mathrm{A} . \mathrm{m}^{-1}\right)$ and $H$ the applied magnetic field $\left(\mathrm{A} . \mathrm{m}^{-1}\right)$ : lengths $L=l \sqrt{A / K}$, magnetization $M_{\mathrm{s}}=\sqrt{K / \mu_{0}} m$, magnetic field $H=\sqrt{K / \mu_{0}} h=\left(2 K / \mu_{0} M_{\mathrm{s}}\right) \tilde{h}$. Notice that we make use of two ways to obtain dimensionless units for magnetic fields, $h$ and $\tilde{h}$. The first one, $h$, is more convenient when it is needed to express dipolar fields, because then $m$ and $h$ play symmetrical roles [see Eq.(6)]. The second one, $\tilde{h}$, is more convenient for discussing magnetization reversal because $\tilde{h}=-1$ is the value for the Stoner-Wohlfarth model. We call Bloch wall width and exchange length the quantities $\Lambda_{\mathrm{Bl}}=\pi \sqrt{A / K}$ and $\Lambda_{\mathrm{ex}}=\sqrt{2 A / \mu_{0} M_{\mathrm{s}}^{2}} \cdot \Lambda_{\mathrm{Bl}}$. and $\pi \Lambda_{\text {ex. }}$ are the length scales over which one may expect a magnetization rotation of roughly $\pi$ under the influence of anisotropy versus exchange, or exchange versus dipolar energy, respectively. Thus, in reduced units, $\lambda_{\mathrm{Bl}}=\pi$ and $\lambda_{\mathrm{ex} .}=\sqrt{2} / m$. Notice that walls are of Néel type in the range of thickness considered. However for the thinnest dots self-dipolar energy becomes negligibly small because of the reduction of dimensionality, so that $\lambda_{\mathrm{Bl}}$. is adequate for describing the Néel wall width, at least in the case of sufficiently large anisotropy. In the case of in-plane uniaxial anisotropy the microscopic energy in the dot is written (dipolar energy excluded, see above sub-section)

$$
E_{\mathrm{V}}=K \sin ^{2} \omega+A(\nabla \omega)^{2}-\mu_{0} M_{\mathrm{s}} H \cos \omega
$$

or, in dimensionless units :

$$
\begin{aligned}
e_{\mathrm{V}} & =\sin ^{2} \omega+(\nabla \omega)^{2}-m h \cos \omega \\
& =\sin ^{2} \omega+(\nabla \omega)^{2}-2 \tilde{h} \cos \omega .
\end{aligned}
$$

Finally, the reduced variable for $z$ will be written $u$. Numerical evaluation of the model will often be made for $m=2.81$, a value for which experimental results are available[18].

\subsection{Practical procedure}

In practice our calculation proceeds in three steps:

(1) The micromagnetic configuration $\omega(u)$ inside the slab is determined by the minimization of Eq.2 (Euler's equation), provided that the magnetization rotation at the edge is set to a value fixed a priori, $\omega_{0}=\omega(u=0)$.

(2) The density of edge magnetic pole is then $m \cos \left(\omega_{0}\right)$, from which the dipolar field $h_{\mathrm{d}}(u)$ is calculated everywhere in the dot, leading to the 
evaluation of the microscopic demagnetizing torque acting on magnetization, $\gamma_{\mathrm{d}}(u)$. From this, the integral torque applied at the edge is evaluated: $\Gamma_{\mathrm{d}}=\int_{-\infty}^{0} \gamma_{\mathrm{d}}(u) \mathrm{d} u$. Notice that torque integrals converge rapidly, contrary to energy integrals. Finally, Euler's equation is applied at the edge (Brown's condition), so that magnetization at the edge is also under equilibrium:

$$
\Gamma_{\text {ex. }}+\Gamma_{\mathrm{d}}=0
$$

where $\Gamma_{\text {ex. }}$ is the exchange torque at the edge.

(3) The possible equilibrium values of $\omega_{0}$ are finally determined self-consistently, by solving Eq. (4). Expanding Eq. (4) to the fifth order leads to a binomial equation:

$$
A\left(\frac{\omega_{0}}{2}\right)^{4}+B\left(\frac{\omega_{0}}{2}\right)^{2}+C=0
$$

The expressions of $A, B$ and $C$ are given in [14]. The predictions of the existing model are reproduced in Fig. $2 \mathrm{~b} . \tilde{h}_{\mathrm{r}}$ increases in absolute value with decreasing $t$, up to the Stoner-Wohlfarth prediction $\left|\tilde{h}_{\mathrm{r}}\right|=1$ in the mathematical limit of zero thickness, i.e. in the case of vanishing dipolar fields. The model does not take into account thermal activation.

\section{$3 \quad$ Improved accuracy}

For both existing and present versions of the model $\mathbf{m}$ is assumed to be uniform along the dot thickness, and $\mathbf{h}_{\mathrm{d}}$ is evaluated taking into account the surface magnetic charges only, given by $\sigma=\mathbf{m} . \mathbf{n}=m \cos \omega_{0}$ ( $\mathbf{n}$ is the outward unit vector normal to the dot edge), neglecting volume charges. For simplicity the value of the dipolar field $h_{\mathrm{d}}(x, u)$ in the previous model was taken equal to its value at mid-height $(x=0)$. This induced an overestimation of the $x$-average of $h_{\mathrm{d}}$, especially close to the dot edge that bears the magnetic poles. In the present paper we calculate the exact $x$-averaged dipolar field acting on $\mathbf{m}$. At any $u<0$ inside the dot the $x$-component of $h_{\mathrm{d}}$ has a zero $x$-averaged value and needs therefore not be calculated. For the $u$ component one finds

$$
h_{\mathrm{d}}(x, u)=\frac{\sigma}{2 \pi}\left[\arctan \left(\frac{2 x+t}{2 u}\right)-\arctan \left(\frac{2 x-t}{2 u}\right)\right]
$$

The $x$-averaged value of this $u$-component is:

$$
h_{\mathrm{d}}(u)=\left\langle h_{\mathrm{d}}(x, u)\right\rangle=\frac{1}{t} \int_{-t / 2}^{t / 2} h_{\mathrm{d}}(x, u) \mathrm{d} x .
$$

We then straightforwardly get 


$$
h_{\mathrm{d}}(u)=\frac{\sigma}{\pi} f(u)
$$

In the first version of the model we had $f(u)=\arctan (t / 2 u)$, whereas here:

$$
f(u)=\left[\arctan \left(\frac{t}{u}\right)-\frac{u}{2 t} \ln \left(1+\frac{t^{2}}{u^{2}}\right)\right]
$$

The calculation scheme is then similar to the one reported in detail in Ref.[14]. The total demagnetizing torque is given by

$$
\Gamma_{\mathrm{d}}=\int_{-\infty}^{0} \gamma_{\mathrm{d}}(u) \mathrm{d} u
$$

with

$$
\gamma_{\mathrm{d}}=-\frac{m^{2} \cos \omega_{0}}{\pi} \sin (\omega) f(u)
$$

After expanding $\gamma_{\mathrm{d}}(u)$ up to the fifth order in terms of $\omega_{0}$ and proceeding to integration we find the same type of formula as in [14]:

$$
\begin{aligned}
\Gamma_{\mathrm{d}}= & \frac{4 m^{2}}{\pi}\left\{\frac{\mathrm{J}_{1}}{2}\left(\frac{\omega_{0}}{2}\right)\right. \\
& +\left[-\frac{1}{6}\left(5 \mathrm{~J}_{1}+3 \mathrm{~J}_{3}\right)+\frac{\alpha^{2}}{8}\left(\mathrm{~J}_{1}-\mathrm{J}_{3}\right)\right]\left(\frac{\omega_{0}}{2}\right)^{3} \\
& +\left[\frac{1}{30}\left(2 \mathrm{~J}_{1}+15 \mathrm{~J}_{3}+15 \mathrm{~J}_{5}\right)+\frac{\alpha^{2}}{8}\left(-\mathrm{J}_{1}-2 \mathrm{~J}_{3}+3 \mathrm{~J}_{5}\right)\right. \\
& \left.+\frac{\alpha^{4}}{32}\left(2 \mathrm{~J}_{1}-3 \mathrm{~J}_{3}+\mathrm{J}_{5}\right)\right]\left(\frac{\omega_{0}}{2}\right)^{5} \\
& \left.+O\left(\omega_{0}\right)^{7}\right\}
\end{aligned}
$$

with

$$
\mathrm{J}_{k}=-\int_{-\infty}^{0} e^{\mu_{k} u} f(u) \mathrm{d} u
$$

and with $\mu_{k}=k \sqrt{1+\tilde{h}}$. The $\mathrm{J}_{k}$ integrals differ from the $\mathrm{I}_{k}$ of Ref.[14], due to the new expression of $f(u)$. After integration we find 


$$
\mathrm{J}_{k}=\frac{1}{\mu_{k}}\left\{\frac{\pi}{2}+\frac{1}{t \mu_{k}}\left[\operatorname{ci}\left(t \mu_{k}\right) \cos \left(t \mu_{k}\right)+\operatorname{si}\left(t \mu_{k}\right) \sin \left(t \mu_{k}\right)-\mathrm{C}-\ln \left(t \mu_{k}\right)\right]\right\}
$$

where $\mathrm{C}$ is Euler's constant, and ci and si are cosine and sine integral functions respectively $[19,14]$. The equilibrium states are found by searching for equilibrium positions of the edge magnetization. These are (in addition to the trivial state $\omega_{0}=0$ ) solutions of Eq. (5) where $A, B$, and $C$ are exactly the same as in [14], replacing the $\mathrm{I}_{k}$ integrals by the new $\mathrm{J}_{k}$. Equation (5) has two roots

$$
\Omega_{0}^{ \pm}=\left(\frac{\omega_{0}^{ \pm}}{2}\right)^{2}=\frac{-B \pm \sqrt{\Delta}}{2 A} .
$$

$\omega_{0}$ is arbitrarily chosen positive, so there will be at most three equilibrium states in the non-reversed case:

- For $\Delta>0$ and $\Omega_{0}^{-}>0$ there are three equilibrium solutions: $0, \omega_{0}^{-}$and $\omega_{0}^{+}$ with $\omega_{0}^{+}>\omega_{0}^{-}>0$. The only stable solution is $\omega_{0}^{-}$and the configuration of the slab is non-uniform (NU) close to the edge.

- For $\Delta>0, \Omega_{0}^{-}<0, \Omega_{0}^{+}>0$ there are two equilibrium solutions: $\omega_{0}=0$ and $\omega_{0}^{+}>0$. The stable solution is a single domain (SD): $\omega^{\text {stable }}(u)=\omega_{0}=0 \forall u$.

- For $\Delta<0$, or $\left(\Delta>0\right.$ and $\left.\Omega_{0}^{ \pm}<0\right)$ there is a single equilibrium solution $\omega_{0}=0$, that is unstable, which means that the magnetization has already reversed.

Notice that there is at most one stable equilibrium state (Fig.2). We define the reversal field $\tilde{h}_{\mathrm{r}}$ as the field for which the stable state vanishes, triggering an irreversible magnetization jump. $\tilde{h}_{\mathrm{r}}$ is determined by $\omega_{0}^{-}$(stable) $=$ $\omega_{0}^{+}$(unstable) in the NU case, and by $\omega_{0}^{+}$(unstable) $=0$ (stable) in the SD state. In the NU case we determine the nucleating field $\tilde{h}_{\mathrm{n}}$ with $\omega_{0}^{-}=0$.

Fig.2a shows the stable and unstable equilibrium states against applied field, for several thicknesses $t$, predicted by the present model. The results are qualitatively similar for both versions. Below a critical thickness $\left(t_{\mathrm{c}} \simeq 0.273\right)$ the magnetization is uniform under any applied field, and the magnetization reversal is abrupt going directly from the SD state antiparallel to the applied field, to the SD state parallel to the applied field. For thicker films $\left(t>t_{\mathrm{c}}\right)$ the magnetization experiences a stable NU state before the abrupt reversal. The transition from the antiparallel SD state to the NU state is of second order. $\omega_{0}$ changes continuously and reversibly from zero to positive values at $\tilde{h}_{\mathrm{n}}$. Similar results are obtained for all $m$ values.

Fig. $2 \mathrm{~b}$ shows the values of $\tilde{h}_{\mathrm{r}}$ and $\tilde{h}_{\mathrm{n}}$ predicted by the existing model, by the 
present improved version, and by numerical calculation performed on a semiinfinite dot without approximations, and so used as a reference. As expected, the original model overestimates the dipolar field so that $\tilde{h}_{\mathrm{r}}$ and $\tilde{h}_{\mathrm{n}}$ have greater values. The new model allows one to reduce the discrepancy with micromagnetic simulations by about $30 \%$.

\section{Model's extension to tilted edges}

We now extend the model to the case where the semi-infinite dot edge is tilted in-the-plane by an angle $\beta$ with respect to the easy axis of magnetization (Fig.3) ( $\beta=90^{\circ}$ previously). The field is still applied parallel to the in-plane anisotropy axis and the dipolar field is calculated using the improved accuracy formulas described above (Eq.8-9). The edge magnetic pole density is now $\sigma=m \sin \left(\beta-\omega_{0}\right)$. We have

$$
\Gamma_{\mathrm{d}}=\frac{m^{2} \sin \left(\beta-\omega_{0}\right)}{\pi} \times\left\{-\int_{-\infty}^{0} f(u) \cos [\beta-\omega(u)] \mathrm{d} u\right\}
$$

where $f$ is defined by equation (9). After some calculation we find: 


$$
\begin{aligned}
\Gamma_{\mathrm{d}} & =\frac{m^{2}}{\pi}\left\{\frac{1}{2} \mathrm{~J}_{0} \sin (2 \beta)\right. \\
+ & \left(\frac{\omega_{0}}{2}\right)\left[2\left(\mathrm{~J}_{1} \sin ^{2} \beta-\mathrm{J}_{0} \cos ^{2} \beta\right)\right] \\
- & \left(\frac{\omega_{0}}{2}\right)^{2} \sin (2 \beta)\left[\mathrm{J}_{2}+2 \mathrm{~J}_{1}+\mathrm{J}_{0}\right] \\
+ & \left(\frac{\omega_{0}}{2}\right)^{3}\left[2\left(\frac{1}{3}+\frac{\alpha^{2}}{4}\right)\left(\mathrm{J}_{1} \sin ^{2} \beta-\mathrm{J}_{0} \cos ^{2} \beta\right)\right. \\
& \left.+\frac{\alpha^{2}}{2}\left(1+\frac{4}{\alpha^{2}}\right)\left(-\mathrm{J}_{3} \sin ^{2} \beta+\mathrm{J}_{0} \cos ^{2} \beta\right)+4\left(\mathrm{~J}_{2} \cos ^{2} \beta-\mathrm{J}_{1} \sin ^{2} \beta\right)\right] \\
+ & \left(\frac{\omega_{0}}{2}\right)^{4} \sin (2 \beta)\left[-2\left(\frac{1}{3}+\frac{\alpha^{2}}{4}\right)\left(\mathrm{J}_{2}+2 \mathrm{~J}_{1}+\mathrm{J}_{0}\right)+\right. \\
& \left.+\left(\frac{\alpha^{2}}{2}+1\right)\left(\mathrm{J}_{4}+\mathrm{J}_{0}\right)+\left(\frac{\alpha^{2}}{2}+2\right)\left(\mathrm{J}_{3}+\mathrm{J}_{1}\right)+2 \mathrm{~J}_{2}\right] \\
+ & \left(\frac{\omega_{0}}{2}\right)^{5}\left[4\left(\frac{1}{15}+\frac{\alpha^{2}}{8}+\frac{\alpha^{4}}{16}\right)\left(\mathrm{J}_{1} \sin ^{2} \beta-\mathrm{J}_{0} \cos ^{2} \beta\right)+\right. \\
& +\frac{\alpha^{2}}{2}\left(1+\frac{3 \alpha^{2}}{4}\right)\left(1+\frac{4}{\alpha^{2}}\right)\left(-\mathrm{J}_{3} \sin ^{2} \beta+\mathrm{J}_{0} \cos ^{2} \beta\right)+ \\
& +4\left(1+\frac{3 \alpha^{2}}{4}\right)\left(\mathrm{J}_{2} \cos ^{2} \beta-\mathrm{J}_{1} \sin ^{2} \beta\right) \\
& +\left(\frac{\alpha^{2}}{8}+\frac{3 \alpha^{2}}{2}+2\right)\left(1+\frac{4}{\alpha^{2}}\right)\left(\mathrm{J}_{3} \sin ^{2} \beta-\mathrm{J}_{2} \cos ^{2} \beta-\mathrm{J}_{0} \cos ^{2} \beta\right)+ \\
& \left.+\frac{2}{\alpha^{2}}\right)\left(-\mathrm{J}_{4} \cos ^{2} \beta+\mathrm{J}_{1} \sin ^{2} \beta\right)
\end{aligned}
$$

The equilibrium states are roots of a fifth order polynomial equation

$$
A_{0}+A_{1}\left(\frac{\omega_{0}}{2}\right)+A_{2}\left(\frac{\omega_{0}}{2}\right)^{2}+A_{3}\left(\frac{\omega_{0}}{2}\right)^{3}+A_{4}\left(\frac{\omega_{0}}{2}\right)^{4}+A_{5}\left(\frac{\omega_{0}}{2}\right)^{5}=0 .
$$

The coefficients $A_{i}$ are functions of $m, \tilde{h}$ and $\mathrm{J}_{k}$ as defined in Eq.(13-14). For $\beta \neq 90^{\circ} \mathbf{h}_{\mathrm{d}}$ is no more parallel to the easy axis of magnetization, so that even far away from the edge $\mathbf{h}_{\mathrm{d}}$ is not mostly antiparallel to $\mathbf{m}$. Consequently the local demagnetizing torque $\boldsymbol{\gamma}_{\mathrm{d}}(u)=\mathbf{m} \wedge \mathbf{h}_{\mathrm{d}}$ decays like $1 / u$ far away from the edge, so that the integrated demagnetizing torque defined mathematically by Eq.(16) diverges. Looking at Eq. (13) it is readily seen that all $\mathrm{J}_{k}$ converge rapidly for $k>0$, whereas $\mathrm{J}_{0}$ diverges logarithmically. This problem is not physical and stems from the edge-torque approximation. To lift the nonphysical divergence of $\Gamma_{\mathrm{d}}$ we introduce a cutoff length $u_{\mathrm{c}}$ to evaluate $\mathrm{J}_{0}$. The value of $u_{\mathrm{c}}$ that one should use to yield physically sound results was estimated the following way. In the case $\beta=90^{\circ}$ the formula for $\Gamma_{d}$ does not make use of 
$\mathrm{J}_{0}$, and therefore converges. The suitable value of $u_{\mathrm{c}}$ was taken as the length for which results integrated numerically with cutoff $u_{\mathrm{c}}$ nearly coincide with exact results [Eq. (5), i.e. with no cutoff]. This procedure yields approximately $u_{\mathrm{c}}=15 \lambda_{\text {ex. }}$ nearly independently of the dot thickness. Notice that, due to the slow logarithmic divergence of $\mathrm{J}_{0}$ the use of a slightly overestimated $u_{\mathrm{c}}$ yields nearly unchanged results. Therefore the results of the model do not critically depend on the exact value of $u_{\mathrm{c}}$ provided that $u_{\mathrm{c}}>15 \lambda_{\text {ex. }}$, a physically meaningful order of magnitude. Let us finally note that numerical integration with increasing values of $u_{\mathrm{c}}$ until results become stationary is an alternative procedure for the determination of $u_{\mathrm{c}}$. Contrary to the procedure described above, this alternative procedure can be applied with arbitrary edge orientations $\beta$, and revealed that $u_{\mathrm{c}}$ is also nearly independent of $\beta$. Accordingly in all results shown below we have used $u_{\mathrm{c}}=15 \lambda_{\text {ex. }}$ and evaluated $\mathrm{J}_{0}$ from expression

$$
\mathrm{J}_{0}=-\int_{-u_{\mathrm{c}}}^{0} f(u) \mathrm{d} u=t\left[\frac{3}{4}+\frac{1}{4} \ln \left(1+\frac{u_{\mathrm{c}}^{2}}{t^{2}}\right)\right] .
$$

The equilibrium states $\omega_{0}$ were determined by solving numerically Eq.(18). The advantage of the analytical expansion is that numerical calculation is reduced to solving a fifth-order equation, avoiding numerical evaluation of integrals or even full micromagnetic calculations using Landau-Lifschitz-Gilbert equations.

\section{Numerical evaluation}

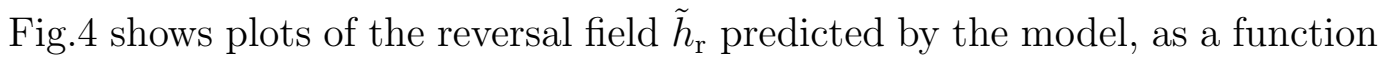
of both edge orientation $\beta$ and dot thickness $t$. We remind the reader that the field is always applied along the anisotropy axis. As expected from the increasing strength of dipolar fields for increasing thickness $t$, for any given edge orientation $\left|\tilde{h}_{\mathrm{r}}\right|$ decreases with increasing $t$. Let us examine the edge orientation dependence of $\left|\tilde{h}_{\mathrm{r}}\right|$. For any given $t$ the reversal field $\left|\tilde{h}_{\mathrm{r}}\right|$ is maximum for $\beta=0^{\circ}$ and equals 1 (the anisotropy field in dimensionless units). Let us give a simple picture for this case. For $\beta=0^{\circ}$ the anisotropy axis lies parallel to the dot edge, so that uniform magnetization is favored and no magnetic poles arise, yielding zero dipolar field. Thus only Zeeman and anisotropy energies compete, explaining that the value $\tilde{h}_{\mathrm{r}}=-1$ expected for coherent rotation with the field applied along the easy axis[15,16] is retrieved. The full edge orientation dependence is more complex. For low thickness a local maximum of $\left|\tilde{h}_{\mathrm{r}}\right|$ associated with a cusp is observed at $\beta=90^{\circ}$, while a smooth absolute minimum is reached for a thickness-dependent intermediate angle. This curve displays similarities with the well-known Stoner-Wohlfarth Astroid [20], although in the present case only a fraction of the magnetic field is applies at 
an angle with the easy axis direction (the dipolar field), whereas the external field remains parallel to the easy axis direction. The similarity with the StonerWohlfarth Astroid is therefore only qualitative. For increasing thickness the absolute minimum for $\left|\tilde{h}_{\mathrm{r}}\right|$ is displaced towards $\beta=90^{\circ}$, finally replacing the cusped local maximum above a critical thickness around 0.35 , slightly higher than the critical thickness $t_{\mathrm{c}}=0.273$. It is not clear whether the similarity between these two figures is a coincidence or not.

Let us now examine the details of the nucleation volumes. Fig.5 shows the direction of the edge magnetization just before the reversal, $\omega_{0}^{\mathrm{r}}$ as a function of the edge direction $\beta$. For low $\beta$ the model predictions come close to the line of slope $1\left(\omega_{0}^{\mathrm{r}}=\beta\right)$, which means that the edge magnetization lies nearly parallel to the edge. This can be understood as this significantly reduces $\Gamma_{d}$ at the moderate expense of exchange energy. For higher $\beta$ the cost of exchange energy is too high so that the magnetization never comes parallel to the edge. Finally Fig. 6 shows the magnetization profile inwards from the dot's edge, as a function of applied field.

\section{Approximate scaling law for the reversal field}

Although the computation of the results does not require special skills, typing Eq. (17) and solving Eq. (18) might rebuke some readers. We therefore propose an approximate polynomial scaling law that empirically fits the variation of $\tilde{h}_{\mathrm{r}}(m, t, \beta)$ :

$$
\log \left(1+\tilde{h}_{\mathrm{r}}\right)=a(m, \beta)+b(m, \beta) \log (t)+c(m, \beta) \log ^{2}(t)
$$

where $a(m, \beta), b(m, \beta)$ and $c(m, \beta)$ are polynomial functions of $m$ and $\beta$ :

$$
\begin{aligned}
& a(m, \beta)=a_{0}(m)+a_{1}(m) \beta+a_{2}(m) \beta^{2} \\
& b(m, \beta)=b_{0}(m)+b_{1}(m) \beta+b_{2}(m) \beta^{2} \\
& c(m, \beta)=c_{0}(m)+c_{1}(m) \beta+c_{2}(m) \beta^{2}
\end{aligned}
$$

and in turn $a_{i}(m), b_{i}(m)$ and $c_{i}(m)(i \in\{0,1,2\})$ are polynomial functions of $m$ :

$$
a_{i}(m)=a_{i 0}+a_{i 1} m+a_{i 2} m^{2}
$$


Coefficients $a_{i j}, b_{i j}$ and $c_{i j}(i, j \in\{0,1,2\})$ are summarized in the three following matrices, respectively, with $\beta$ in degrees.

$$
\begin{gathered}
{\left[a_{i j}\right]=\left(\begin{array}{rrr}
-1.156840 & -0.886400 & 0.094000 \\
0.019510 & 0.018440 & -0.002300 \\
-0.000027 & -0.000135 & 0.000017
\end{array}\right)} \\
{\left[b_{i j}\right]=\left(\begin{array}{rrr}
1.161640 & -1.159410 & 0.152820 \\
-0.025000 & 0.022940 & -0.003410 \\
0.000561 & -0.000298 & 0.000036
\end{array}\right)} \\
{\left[c_{i j}\right]=\left(\begin{array}{rrr}
0.005700 & -0.270650 & 0.046350 \\
-0.008360 & 0.007290 & -0.001180 \\
0.000190 & -0.000114 & 0.000014
\end{array}\right)}
\end{gathered}
$$

These figures yield the best fit to the model's exact result in the range $2.4 \leq$ $m \leq 6.0, t<0.4$ and any inclination $\beta$. The accuracy of the scaling law is in most cases better than $1 \%$, except for $\beta$ values close to $90^{\circ}$ where the error reaches $4 \%$ at most.

\section{Comparison with LLG numerical calculation and discussion}

\subsection{Numerical protocol}

In the following we use the results of simulations as a reference for assessing the validity of the model. Numerical calculations were performed by integrating the Landau-Lifschitz-Gilbert equation in the framework of finite volumes with prismatic cells[21], with a mesh size of $5 \AA$, under quasi-static conditions from positive saturation to negative fields and finally magnetization reversal. We used periodic boundary conditions along the $y$ direction to simulate a laterally infinite stripe. The width $w$ of the stripe (i.e. along $u$ ) was varied from $100 \mathrm{~nm}$ to $500 \mathrm{~nm}$. A $1 / w^{2}$ scaling law was then found to be suitable to extrapolate the results to infinite $w$, for comparison with the model describing a half-infinite stripe. Finally the values of $\tilde{h}_{\mathrm{r}}$ and edge magnetization direction at $\tilde{h}_{\mathrm{r}}, \omega_{0}^{\mathrm{r}}$, were

refined using an inverse squared susceptibility scaling law: $\chi \sim 1 / \sqrt{\tilde{h}-\tilde{h}_{\mathrm{r}}}$ $[22,14]$. This allowed us to reduce effects of the finite field step used in the simulation. 


\subsection{Comparison of reversal field values}

For all $\beta$ we find a good agreement for $\left|\tilde{h}_{\mathrm{r}}\right|$ values predicted by the model and the simulations (Fig.7). The agreement is better for lower thickness. As the geometry of the model (approximation 2) is identical to that of the simulation this good agreement proves that approximations 1) and 3) used in the model are physically sound. In particular this shows that concentrating all demagnetizing effects at the dot's edge (edge torque, approximation 3) is physically relevant in the case of thin flat dots made of a magnetically-hard material, whatever the edge orientation is. This extends the conclusion of [14], and further confirms the relevance of the concept of edge torque first discussed in $[22]$.

\subsection{Range of validity of the model}

We noticed above that the agreement of simulations with the model is better for thinner structures. This was expected from the model's three approximations proposed in section 2.1, that were justified by the small thickness. In this paragraph we propose quantitative arguments to assess the range of validity of these approximations, in terms of thickness and anisotropy magnitude.

In order to satisfy approximation 1 the magnetization shall not be free to vary over a distance $t$ along the normal to the dot surface. $t$ must therefore be reasonably smaller than $\Lambda_{\mathrm{Bl}}$. and $\pi \Lambda_{\text {ex. }}$. Thus two necessary relationships are: $t \lesssim \sqrt{A / K}$ and $t \lesssim \sqrt{2 A / \mu_{0} M_{\mathrm{s}}^{2}}$.

In order to satisfy approximation 2 the length of the edge considered must be significantly greater than $\Lambda_{\mathrm{Bl}}$. and $\pi \Lambda_{\text {ex. }}$.

Approximation 3 holds two aspects. The first aspect is that nucleation volumes must be large compared to the close-to-the-edge area where demagnetizing fields are non-negligible. Thus, viewing nucleation volumes as a portion of wall, the required condition is again $\sqrt{A / K} \gtrsim t$. The second aspect is to neglect volume charges, so that the shape of nucleation volumes can be approximated by a portion of wall. Neglecting the influence of the applied field on the internal rigidity of the wall, this requirement is equivalent to the anisotropy field $2 K / \mu_{0} M_{\mathrm{s}}$ being large compared to demagnetizing fields $H_{\mathrm{d}}$ arising from volume charges. The lateral range of dipolar fields is $t$, so that $H_{\mathrm{d}}$ at a given point $\mathrm{P}$ is roughly the sum of dipolar fields $H_{\mathrm{d}, i}$ and $H_{\mathrm{d}, i+1}$ arising from two horizontal cylinders of square section $t \times t$ located on either side of P, labelled $i$ and $i+1$ on Fig.9. The density of volume charges scales like $\operatorname{div}\left(\mathbf{M}_{\mathrm{s}}\right) \sim M_{\mathrm{s}} \frac{\partial \omega}{\partial z} \sim M_{\mathrm{s}} \sqrt{K / A}$, so that $H_{\mathrm{d}, i} \sim M_{\mathrm{s}} t \sqrt{K / A}$. Due to the opposite 
signs of $H_{\mathrm{d}, i}$ and $H_{\mathrm{d}, i+1}$, for their sum one shall not consider $\operatorname{div}\left(\mathbf{M}_{\mathrm{s}}\right)$ but instead its variation over distance $t$, i.e. $t \frac{\partial}{\partial z}\left(M_{\mathrm{s}} \frac{\partial \omega}{\partial z}\right) \sim M_{\mathrm{s}} \frac{\partial^{2} \omega}{\partial z^{2}} t \sim M_{\mathrm{s}} t(K / A)$. Thus, $H_{\mathrm{d} \text {,tot }} \sim M_{\mathrm{s}} t^{2}(K / A)$. When compared to the anisotropy field, this again yields criterium $t \lesssim \Lambda_{\text {ex. }}$.

To conclude, the conditions for confident validity of the model are twofold:

(1) The size of the dot or stripe and that of its edges must be much larger than both $\Lambda_{\mathrm{Bl}}=\pi \sqrt{A / K}$ and $\pi \Lambda_{\mathrm{ex} .}=\pi \sqrt{2 A / \mu_{0} M_{\mathrm{s}}^{2}}$.

(2) The thickness $t$ of the dot or stripe must be equal or smaller than $\sqrt{A / K}$ and $\sqrt{2 A / \mu_{0} M_{\mathrm{s}}^{2}}$.

Notice that the model shall however not be applicable to very soft materials. This does not show explicitly in the above criteria because we did not consider logarithmic tails of Néel walls, that become important for soft materials.

\subsection{Consequences of the approximation of the edge torque}

We have seen in the previous subsections that the approximation of the edge torque is physically relevant to predict global quantities like the value of the reversal field. In this paragraph we show that microscopic details of the magnetization state might differ, however not preventing the correct prediction of global quantities. This finding confirms suggestions of Rave et al. about the concept of edge torque[23]. Fig. 8 shows calculated and simulated curves for $\omega_{0}^{\mathrm{r}}(\beta)$, as well as magnetization profiles $\omega(u)$ just before magnetization reversal. Regarding the angular variation of $\omega_{0}^{\mathrm{r}}(\beta)$, we find a satisfactory qualitative agreement between model and simulations. However, $\omega_{0}^{\mathrm{r}}(\beta)$ predicted by the model is always larger than that given by simulation (see Fig.8). This presumably results from the torque approximation that concentrates all demagnetizing effects at the edge. A further consequence of this approximation is revealed by examining magnetization profilesé $\omega(u)$. Again, we observe a reasonable qualitative agreement between model and simulations. However the characteristic length scale (e.g. the half-width of the non-uniform region) is slightly larger in simulations. This is related to the volume charges being neglected in the model, and all demagnetizing effects being rejected at the edge. This confirms that it is not necessary for micromagnetics to describe the details of the magnetization state close to edges and corners on a length scale much smaller than $\Lambda_{\text {ex. }}$ and $\Lambda_{\mathrm{Bl}}$. to yield correct values for reversal fields[23]. 


\subsection{Implications for experiments}

The model is suitable for describing dots and stripes of various orientations of the edges. From Fig.4 we infer that if a dot has edges of different orientations, each having a sufficient length (see previous sub-section), then magnetization reversal will occurbe initiated along an edge whose associated reversal field is the lowest available among all edges. Whereas this means edges perpendicular to the axis of magnetocrystalline anisotropy for significant thickness $t \gtrsim 0.35$, for $t \lesssim 0.35$, the reversal is not necessarily triggered at edges perpendicular to the easy magnetization axis, but might occur at edges slightly tilted in the range of low thickness. This results from the fact that what triggers the reversal is not directly the strength of magnetic field (external plus demagnetizing), but its torque with magnetization, which implies a compromise on the angle, more precisely between the magnitude of the dipolar field scaling roughly with $\sin \left(\beta-\omega_{0}\right)$, and $\cos [\beta-\omega(u)]$ arising in the torque calculation due and related to the angle between magnetization and $h_{\mathrm{d}}(u)$. Thus, the behavior of stripes and dots made of hard magnetic material is expected to be different from the case of dots made of very soft material, were it is usually admitted that the reversal is triggered along edges that display the highest angle with the mean magnetization direction[13].

\section{Conclusion}

We have proposed an analytical model of magnetization reversal in thin flat dots and stripes with in-plane uniaxial anisotropy, with the external field applied parallel to the anisotropy axis, and relying on suitable approximations. Compared to a previously published article[14] we improved the accuracy by about $30 \%$, and extended the geometry of the dot to an arbitrary in-plane edge orientation. The magnetization configuration and the reversal field $\tilde{h}_{\mathrm{r}}$ are predicted as a function of magnetization, dot thickness and edge orientation. $\left|\tilde{h}_{\mathrm{r}}\right|$ decreases with increasing thickness, due to the increasing demagnetizing effect of internal dipolar fields. For a given thickness the lowest value of $\left|\tilde{h}_{\mathrm{r}}\right|$ is found for edges perpendicular to the anisotropy axis (high thickness range), or at an intermediate angle (low thickness range). $\left|\tilde{h}_{\mathrm{r}}\right|$ reaches the value of coherent rotation, for edges parallel to the anisotropy axis or in the limit of vanishing thickness. An approximate although accurate scaling law is proposed, with a view to allowing one to compute $\tilde{h}_{\mathrm{r}}$ easily. The model is valid for dots and stripes of lateral size much larger (resp. thickness lower) than both exchange length and wall width, and with a non-vanishing uniaxial in-plane magnetocrystalline anisotropy. The reversal field values predicted by the model are found to be in excellent agreement with numerical simulations. 


\section{References}

[1] J. G. Zhu and Y. Zheng, in Spin dynamics in confined magnetic structures, Vol. 83 of Topics in applied physics, edited by B. Hillebrands and K. Ounadjela (Springer, Berlin, 2002), pp. 289-325.

[2] E. Hirota, H. Sakakima, and K. Inomata, in Giant magneto-resistance devices, Vol. 40 of Springer series in surface sciences, edited by G. Ertl, R. Gomer, H. Lüth, and D. L. Mills (Springer, Berlin, 2002), .

[3] R. P. Cowburn, J. Phys. D: Appl. Phys. 33, R1 (2000).

[4] K. J. Kirk, J. N. Chapman, and C. D. W. Wilkinson, J. Appl. Phys. 85, 5237 (1999).

[5] H. Endoa, Y. Uesaka, Y. N. N. Hayashi, and H. Fukushima, J. Appl. Phys. 93, 9865 (2003).

[6] J. G. Deak and R. H. Koch, J. Magn. Magn. Mater. 213, 25 (2000).

[7] R. M. H. New, R. F. W. Pease, and R. L. White, IEEE Trans. Magn. 31, 3805 (1995).

[8] A. Fernandez, M. R. Gibbons, M. A. Wall, and C. J. Cerjan, J. Magn. Magn. Mater. 190, 171 (1998).

[9] J. M.Schneider, H.Hoffmann, J. Magn. Magn. Mater. 257, 1 (2003).

[10] P. Bryant and H. Suhl, Appl. Phys. Lett. 54, 78 (1989).

[11] R. P. Cowburn and M. E. Welland, J. Appl. Phys. 86, 1035 (1999).

[12] K. Y. Guslienko, V. Novosad, Y. Otani, H. Shima, and K. Fukamichi, Appl. Phys. Lett. 78, 3848 (2001).

[13] M. Grimsditch, A. Berger, J. Johnson, V. Metlushko, B. Ilic, P. Neuzil, and R. Kumar, Europhys. Lett. 54, 813 (2001).

[14] O. Fruchart, J.-C. Toussaint, and B. Kevorkian, Phys. Rev. B 63, 174418 (2001).

[15] L. Néel, C. R. Acad. Sci. 224, 1550 (1947).

[16] E. C. Stoner and E. P. Wohlfarth, Phil. Trans. Royal Soc. London A240, 599 (1948).

[17] A. Aharoni, Introduction to the Theory of Ferromagnetism, Vol. 93 of International Series of Monographs on Physics (Oxford University Press, Oxford, 1996).

[18] O. Fruchart, J.-P. Nozières, W. Wernsdorfer, D. Givord, F. Rousseaux, and D. Decanini, Phys. Rev. Lett. 82, 1305 (1999).

[19] I. S. Gradshteyn and I. M. Ryzhik, Table of Integrals, Series, and Products, 5th ed. (Academic Press, London, 1994). 
[20] J. C. Slonczewski, Research Memo RM 003.111.224, IBM Research Center, Poughkeepsie, NY (unpublished).

[21] J. C. Toussaint, A. Marty, N. Vukadinovic, J. Ben Youssef, and M. Labrune, Comput. Mater. Sci. 24, 175 (2002).

[22] A. Hubert and W. Rave, Phys. Stat.Sol. (b) 211, S815 (1999).

[23] W. Rave, K. Ramstöck, and A. Hubert, J. Magn. Magn. Mater. 183, 329 (1998). 


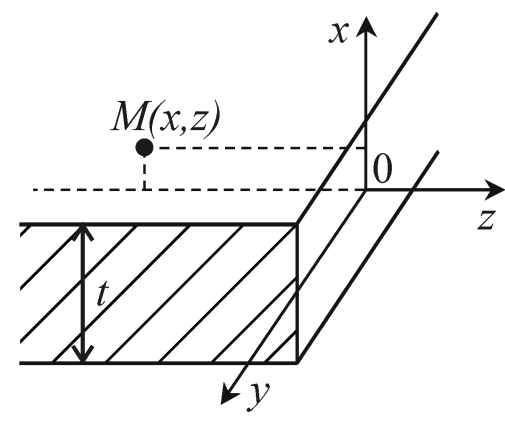

(a)

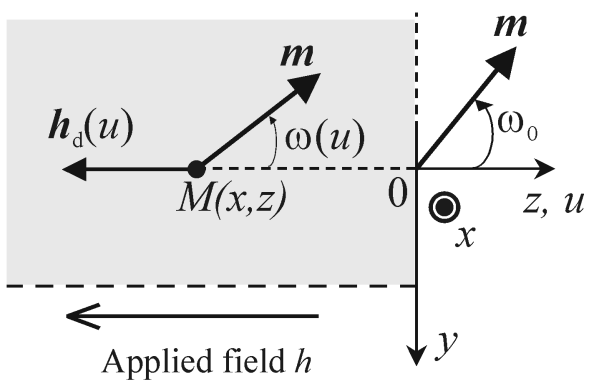

(b)

Fig. 1. (a) Geometry of the model. The semi-infinite slab corresponds to $z<0$, (b) Top view of the dot. $u$ is the dimensionless variable for $z$

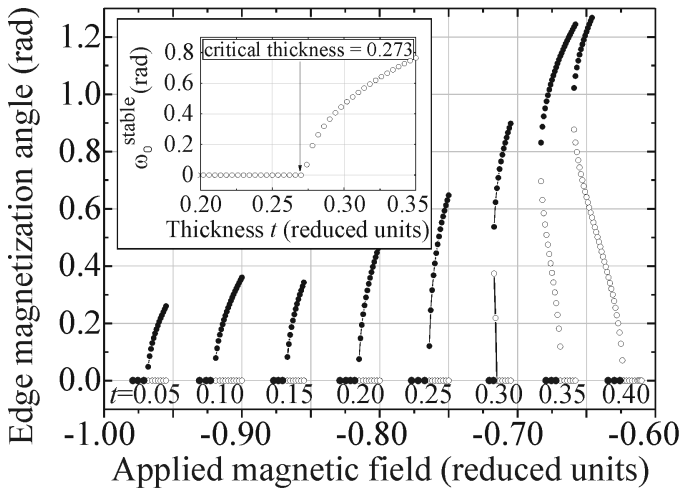

(a)

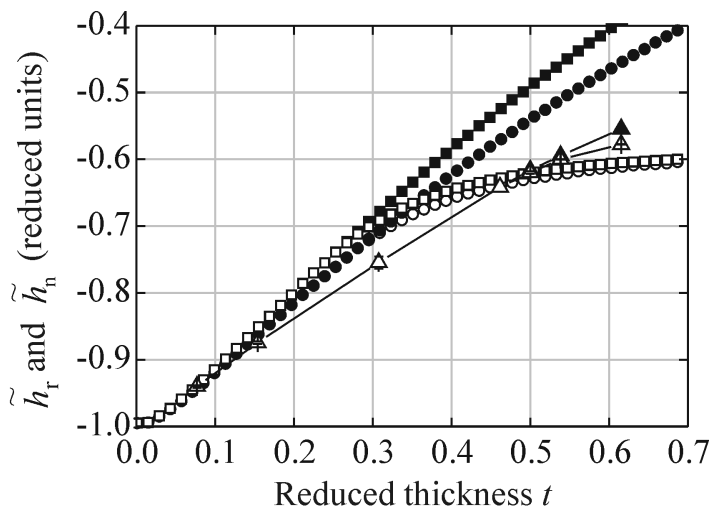

(b)

Fig. 2. (a) Stables (o) and unstable $(\bullet)$ equilibrium states predicted by the present improved model for an edge perpendicular to the anisotropy axis and thicknesses ranging from 0.05 up to 0.40 . Inset: $\omega_{0}^{\mathrm{r}}$ ( $\omega_{0}$ just before reversal), as function of thickness. The transition between SD to NU at the reversal is found for $t_{\mathrm{c}}=0.273$. (b) Nucleation field (full symbols) and reversal field (open symbols), predicted by [14](squares), the present model (disks), and numerical simulations (triangles) [14].

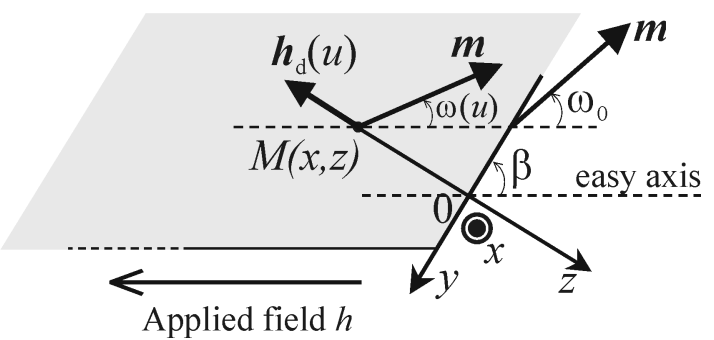

Fig. 3. Top view of the extended geometry of the new model: the edge is tilted by an arbitrary angle $\beta \neq 90^{\circ}$ with respect to the easy axis. 


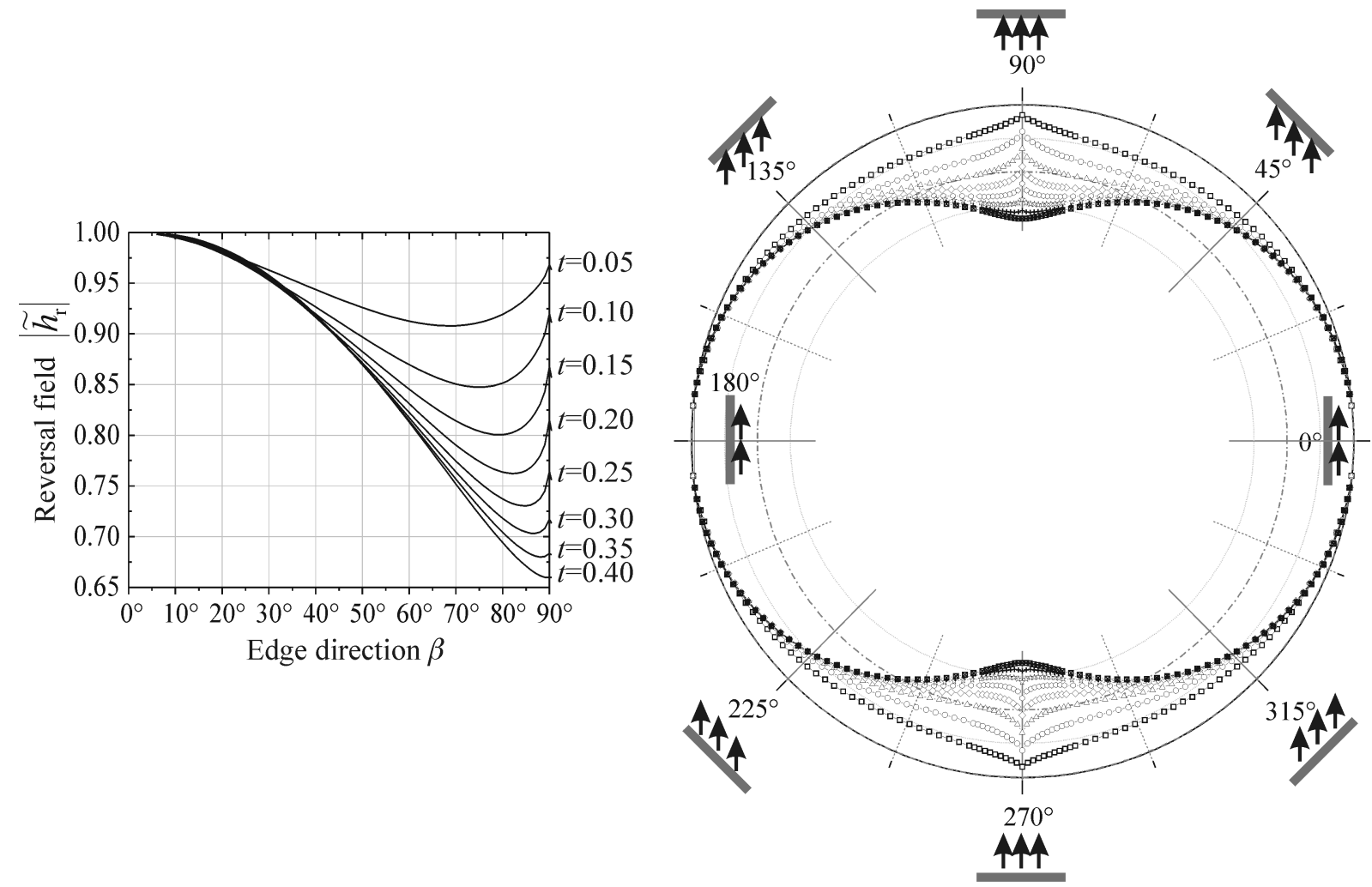

(a)

(b)

Fig. 4. Reversal field $\left|\tilde{h}_{\mathrm{r}}\right|$ predicted by the analytical model as a function of edge orientation $\beta$ for several thicknesses $t$. (a) Cartesian plot, (b) Polar plot: the angle is the edge orientation $\beta$ while the radius stands for $\left|\tilde{h}_{\mathrm{r}}\right|$. The outer diameter stands for $\left|\tilde{h}_{\mathrm{r}}\right|=1$. The orientation of the easy axis with respect to the edge is sketched around the plot.

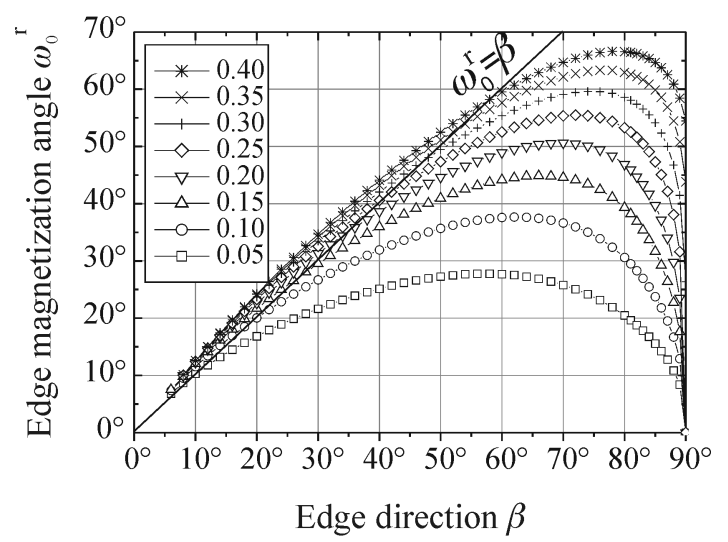

Fig. 5. Edge magnetization angle $\omega_{0}^{\mathrm{r}}$ ( $\omega_{0}$ just before reversal) as a function of edge orientation $\beta$, for thicknesses $t$ ranging from 0.05 up to 0.40 


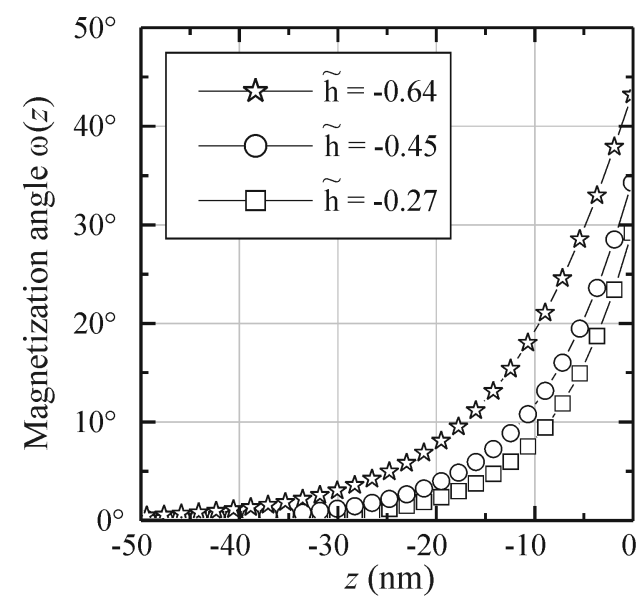

(a)

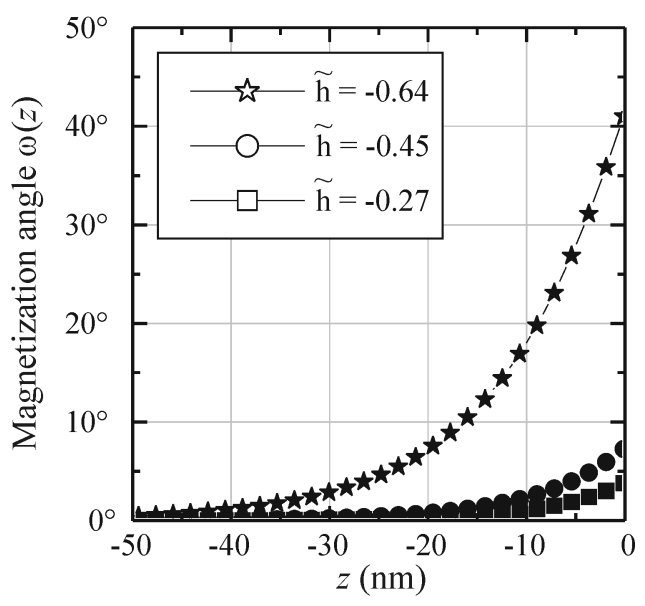

(b)

Fig. 6. Magnetization angle $\omega(z)$ predicted by the model in a dot of thickness $27 \AA$ for several applied magnetic fields: In (a) the edge direction is $\beta=70^{\circ}$ and $\tilde{h}_{\mathrm{r}}=-0.75$, and in (b) $\beta=89^{\circ}$ and $\tilde{h}_{\mathrm{r}}=-0.66$. Physical parameters are: $A=2 \times 10^{-11} \mathrm{~J}_{\mathrm{m}}^{-1}$, $M_{\mathrm{s}}=1.73 \times 10^{6} \mathrm{~A} . \mathrm{m}^{-1}, K=4.76 \times 10^{5} \mathrm{~J} \cdot \mathrm{m}^{-3}$.

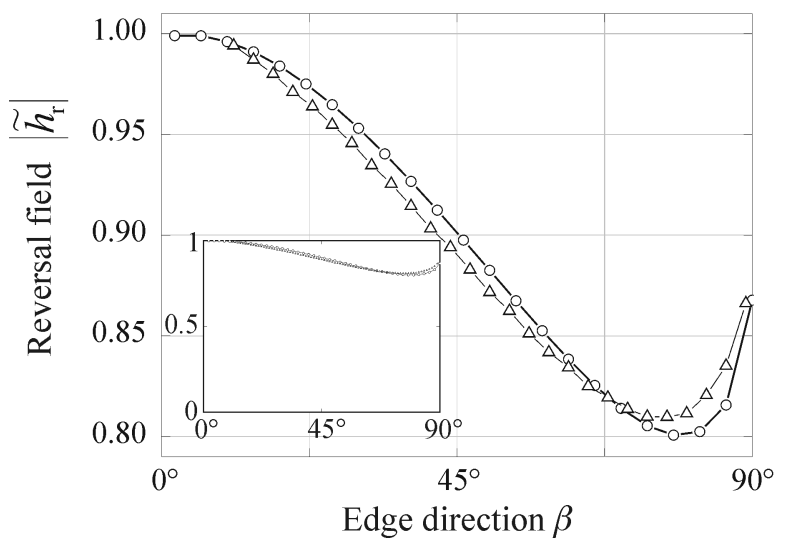

(a)

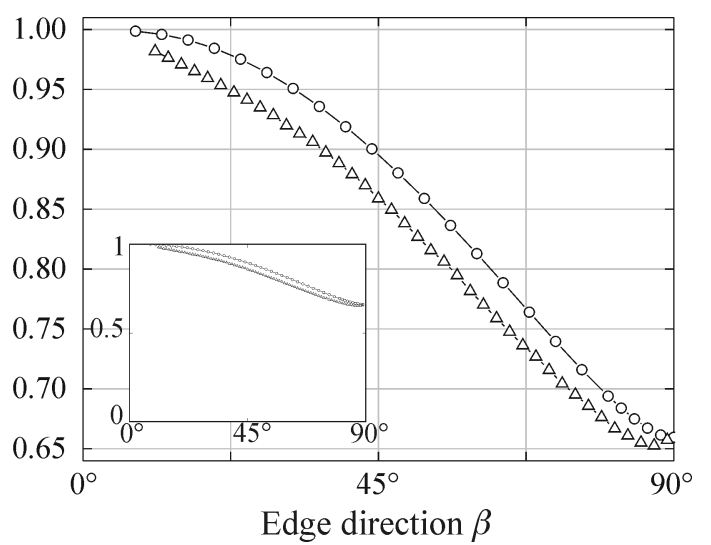

(b)

Fig. 7. Reversal field $\left|\tilde{h}_{\mathrm{r}}\right|$ predicted by the model (o) and numerical simulations $(\triangle)$ for two thicknesses $t=10 \AA$ (a) and $t=27 \AA$ (b). Insets: Same curves but with

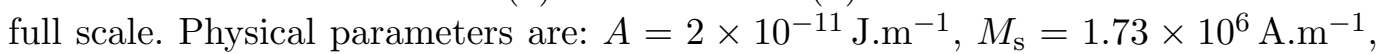
$K=4.76 \times 10^{5}{\mathrm{~J} . \mathrm{m}^{-3}}$. 


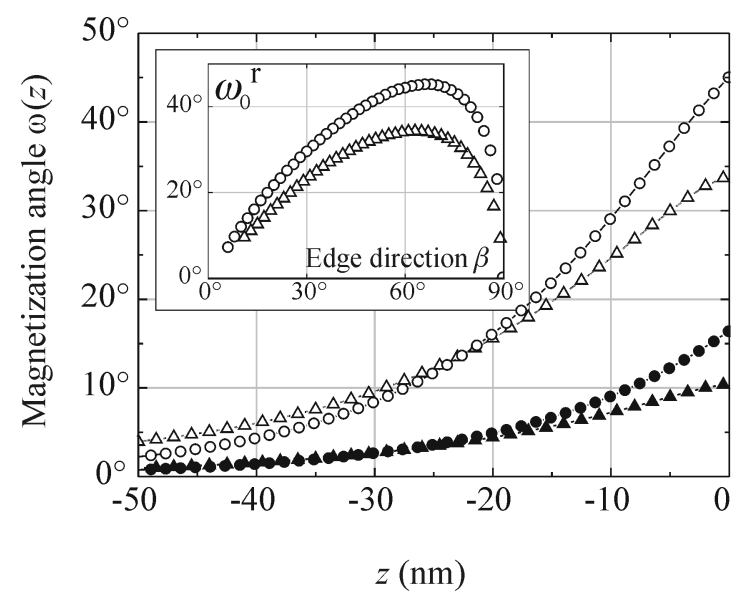

(a)

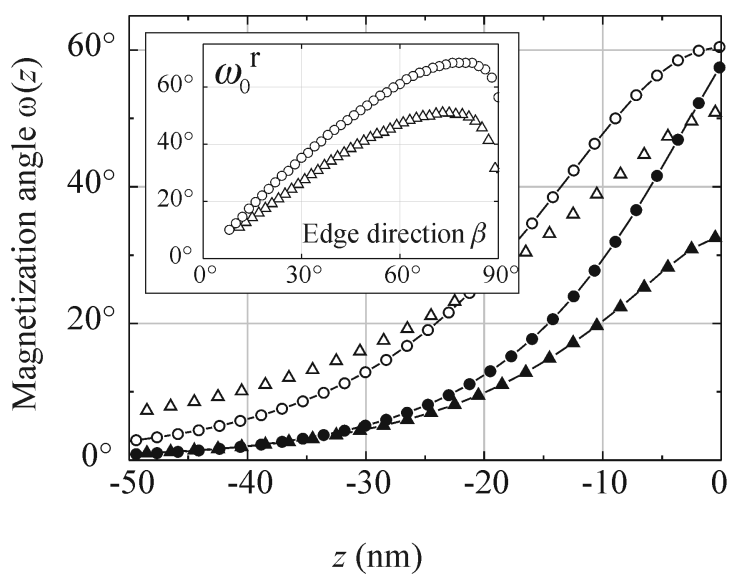

(b)

Fig. 8. Magnetization profile $\omega(u)$ close to the edge, just before magnetization reversal, predicted by the model (disks) and simulation (triangles) for two thicknesses $10 \AA$ (a) and $27 \AA$ (b) and two edge orientations $70^{\circ}$ (open symbols) and $89^{\circ}$ (full symbols). Insets: edge spin angle just before reversal $\omega_{0}^{\mathrm{r}}$ as a function of edge ori-

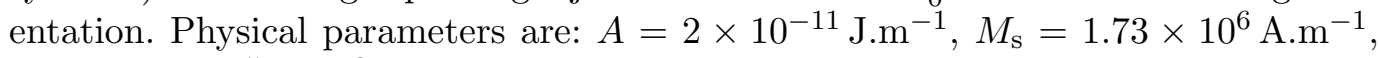
$K=4.76 \times 10^{5}{\mathrm{~J} . \mathrm{m}^{-3}}$.

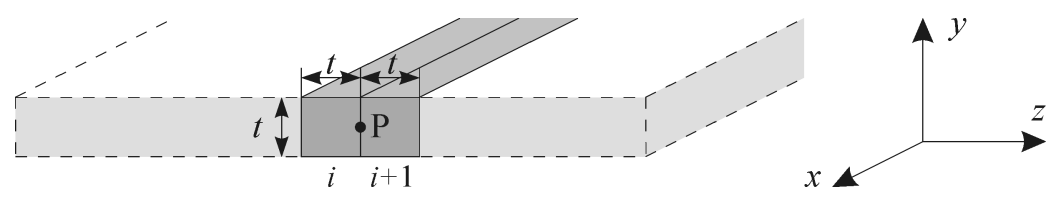

Fig. 9. Schematic illustration of the two in-plane cylinders $i$ and $i+1$ of section $t \times t$ taken into account to estimate the strength of dipolar fields arising from volume magnetic charges. 